\title{
Reducing mitochondrial bound hexokinase II mediates transition from non-injurious into injurious ischemia/reperfusion of the intact heart
}

\author{
Rianne Nederlof ${ }^{1} \cdot$ Ebru Gürel-Gurevin ${ }^{1,2}$ - Otto Eerbeek ${ }^{3}$. Chaoqin $\mathrm{Xie}^{4}$. \\ G. Sjoerd Deijs ${ }^{1}$ - Moritz Konkel ${ }^{1}$ - Jun Hu ${ }^{4}$ • Nina C. Weber ${ }^{1}$ Cees A. Schumacher ${ }^{5}$. \\ Antonius Baartscheer ${ }^{5}$ Egbert G. Mik ${ }^{6}$ Markus W. Hollmann ${ }^{1}$. Fadi G. Akar ${ }^{4}$. \\ Coert J. Zuurbier ${ }^{1}$
}

Received: 25 July 2016 / Accepted: 10 February 2017 / Published online: 3 March 2017

(C) The Author(s) 2017. This article is published with open access at Springerlink.com

\begin{abstract}
Ischemia/reperfusion (I/R) of the heart becomes injurious when duration of the ischemic insult exceeds a certain threshold (approximately $\geq 20 \mathrm{~min}$ ). Mitochondrial bound hexokinase II (mtHKII) protects against I/R injury, with the amount of mtHKII correlating with injury. Here, we examine whether mtHKII can induce the transition from non-injurious to injurious $\mathrm{I} / \mathrm{R}$, by detaching HKII from mitochondria during a non-injurious $\mathrm{I} / \mathrm{R}$ interval. Additionally, we examine possible underlying mechanisms (increased reactive oxygen species (ROS), increased oxygen consumption $\left(\mathrm{MVO}_{2}\right)$ and decreased cardiac energetics) associated with this transition. Langendorff perfused rat
\end{abstract}

Rianne Nederlof and Ebru Gürel Gurevin contributed equally.

Coert J. Zuurbier

c.j.zuurbier@amc.uva.nl

1 Laboratory of Experimental Intensive Care and Anesthesiology (L.E.I.C.A.), Department of Anesthesiology, Academic Medical Center, Meibergdreef 9, 1105 AZ Amsterdam, The Netherlands

2 Department of Biology, Faculty of Science, University of Istanbul, 34134, Fatih, Istanbul, Turkey

3 Department of Physiology, Academic Medical Center, Meibergdreef 9, 1105 AZ Amsterdam, The Netherlands

4 Cardiovascular Research Center, Mt. Sinai School of Medicine, New York, NY 10029, USA

5 Department of Clinical and Experimental Cardiology, Academic Medical Center, Meibergdreef 9, 1105 AZ Amsterdam, The Netherlands

6 Department of Anesthesiology, Laboratory of Experimental Anesthesiology, Erasmus University Medical Center Rotterdam, 's Gravendijkwal 230, 3015 CE Rotterdam, The Netherlands hearts were treated for 20 min with saline, TAT-only or $200 \mathrm{nM}$ TAT-HKII, a peptide that translocates HKII from mitochondria. Then, hearts were exposed to non-injurious 15-min ischemia, followed by 30 -min reperfusion. I/R injury was determined by necrosis (LDH release) and cardiac mechanical recovery. ROS were measured by DHE fluorescence. Changes in cardiac respiratory activity (cardiac $\mathrm{MVO}_{2}$ and efficiency and mitochondrial oxygen tension $\left(\mathrm{mitoPO}_{2}\right)$ using protoporphyrin IX) and cardiac energetics (ATP, PCr, $\Delta \mathrm{G}_{\text {ATP }}$ ) were determined following peptide treatment. When exposed to 15 -min ischemia, control hearts had no necrosis and $85 \%$ recovery of function. Conversely, TAT-HKII treatment resulted in significant LDH release and reduced cardiac recovery $(25 \%)$, indicating injurious $\mathrm{I} / \mathrm{R}$. This was associated with increased ROS during ischemia and reperfusion. TAT-HKII treatment reduced $\mathrm{MVO}_{2}$ and improved energetics (increased $\mathrm{PCr}$ ) before ischemia, without affecting $\mathrm{MVO}_{2} / \mathrm{RPP}$ ratio or mitoPO $\mathrm{O}_{2}$. In conclusion, a reduction in mtHKII turns non-injurious $\mathrm{I} / \mathrm{R}$ into injurious $\mathrm{I} / \mathrm{R}$. Loss of mtHKII was associated with increased ROS during ischemia and reperfusion, but not with increased $\mathrm{MVO}_{2}$ or decreased cardiac energetics before damage occurs.

Keywords Ischemia/reperfusion injury · Hexokinase ·

Reactive oxygen species · Oxygen consumption

\section{Introduction}

The best method to reduce cardiac injury after ischemic heart disease, like a myocardial infarction, is to shorten the ischemic period by quickly restoring reperfusion. However, reperfusion itself can paradoxically cause further injury (ischemia/ 
reperfusion (I/R) injury) and increase infarct size. This can lead to heart failure, which is currently the highest contributor to death caused by cardiovascular disease. If, however, the size of the infarct is decreased, the degree of heart failure will also decrease. Therefore, unravelling the important determinants that dictate when injury starts to develop during $I / R$ is crucial in devising new $\mathrm{I} / \mathrm{R}$ therapies.

Previous research has shown that reduced binding of the glycolytic enzyme hexokinase II (HKII) to the mitochondria (mtHKII) exacerbates the injury of an already injurious I/R insult $[28,33,39]$. It is, however, unknown whether reducing mtHKII during a normally non-injurious I/R episode can transform it into an injurious episode. In that case, the amount of mtHKII is an important determinant of turning reversible, non-injurious I/R into one that is injurious. The underlying mechanisms associated with this transition can then allude to the important processes causing this injury. Proposed physiological mechanisms linking decreased mtHKII to injury are increased reactive oxygen species (ROS) production, increased mitochondrial respiration and decreased high-energy phosphates (phosphocreatine (PCr)) and ATP [7, 22, 25, 28].

Decreasing mtHKII was reported to increase ROS production in isolated cardiac and brain mitochondria [7, 32], in neonatal cardiomyocytes [39] and following an injurious I/R period in the isolated rat heart [25]. Whether loss of mtHKII can already induce ROS production during a much shorter non-injurious period of ischemia in the isolated rat hearts is, however, unknown. It has now become clear that increased activity of the mitochondrial respiratory chain contributes to I/ $\mathrm{R}$ injury $[3,30]$. We have shown that non-injurious ischemia, like ischemic preconditioning (IPC), increases mtHKII [12, 41] and slows down mitochondrial activation [43], but only with glucose present [42], suggesting a necessity for HK. In addition, structurally obstructing HKII attachment to mitochondria obliterated IPC cardioprotection [1]. These findings are commensurate with the glucose dependency of IPC [21]. From these different observations, we hypothesise that decreasing mtHKII may result in increased mitochondrial activity, i.e. oxygen consumption, which then can contribute to increased I/R injury.

Finally, hexokinase binds to the voltage-dependent anion channel (VDAC). VDAC is responsible for the transport of adenine nucleotides and other metabolites across the outer mitochondrial membrane (OMM) [6]. Its close proximity to ATP/ADP exchange can give HKII an important role in cardiac energetics (ATP and PCr). However, it is unknown whether loss of mtHKII in the intact heart before the ischemic period affects cardiac energetics. It is possible that decreasing mtHKII results in impaired cardiac energetics before ischemia, in which impairment may then contribute to the development of injurious I/R. Additionally, when reducing mtHKII does indeed result in injurious $\mathrm{I} / \mathrm{R}$, cardiac energetics after ischemia is then anticipated to be reduced as a consequence of the increased injury (but the decreased energetics can then not be viewed as a driver of augmented I/R injury, because the changes occur after the $\mathrm{I} / \mathrm{R}$ insult).

Therefore, in this study, we tested the hypothesis that decreasing mtHKII mediates the transition from non-injurious towards injurious cardiac $\mathrm{I} / \mathrm{R}$ and that this transition is associated with increased ROS production, decreased $\mathrm{PCr}$ and increased oxygen consumption.

\section{Methods}

\section{Animals}

Animal experiments were approved by the animal Ethical Committee of the University of Amsterdam. Male Wistar rats $(324 \pm 3 \mathrm{~g})$ were obtained from Charles River. Animals were housed 2-4 per cage, fed ad libitum and were subjected to a 12-h dark/12-h light cycle.

\section{Heart perfusion}

The different perfusion protocols are presented in Fig. 1. Rats were anaesthetised with pentobarbital $(60 \mathrm{mg} / \mathrm{kg})$, tracheotomy was performed and mechanical ventilation started. Intravenous heparin (150 IU) was administered after opening of the thorax. The aorta was cannulated in situ, perfusion started and the heart was excised. Hearts were Langendorff perfused at a constant flow (initial perfusion pressure $80 \mathrm{mmHg}$ ) at $37{ }^{\circ} \mathrm{C}$ with Krebs-Henselheit buffer (KHB) containing the following $(\mathrm{mM}): 118 \mathrm{NaCl}, 0.5$ EDTA, 4.7 $\mathrm{KCl}, 2.25 \mathrm{CaCl}_{2}, 1.2 \mathrm{MgSO}_{4}, 25 \mathrm{NaHCO}_{3}, 1.2 \mathrm{KH}_{2} \mathrm{PO}_{4}$ and 11 glucose gassed with $95 \% \mathrm{O}_{2} / 5 \% \mathrm{CO}_{2}$. End-diastolic pressure was set at $\sim 3-5 \mathrm{mmHg}$ using a water-filled polyethylene balloon inserted into the left ventricular cavity. Hearts were continuously submerged in $37^{\circ} \mathrm{C}$ KHB.

\section{Peptide treatment}

After a stabilisation period, hearts were treated with saline (control group 1), $1 \mu \mathrm{M}$ TAT-only (control group 2), $200 \mathrm{nM}$ TAT-HKII or $1 \mu \mathrm{M}$ TAT-HKII. TAT-HKII (MIASHMIACLFTELN( $\beta$-Ala)GYGRKKRRQRRRG-amide) and TAT-only (GYGRKKRRQRRRG-amide) are soluble peptides produced by Pepscan Presto (Lelystad, The Netherlands). TAT-HKII translocates HKII from the mitochondria to the cytosol $[5,25,33]$. Peptides were dissolved in KHB solution and administered through a side-arm connected to a mixing chamber above the heart at $1 \%$ of total perfusion flow. 


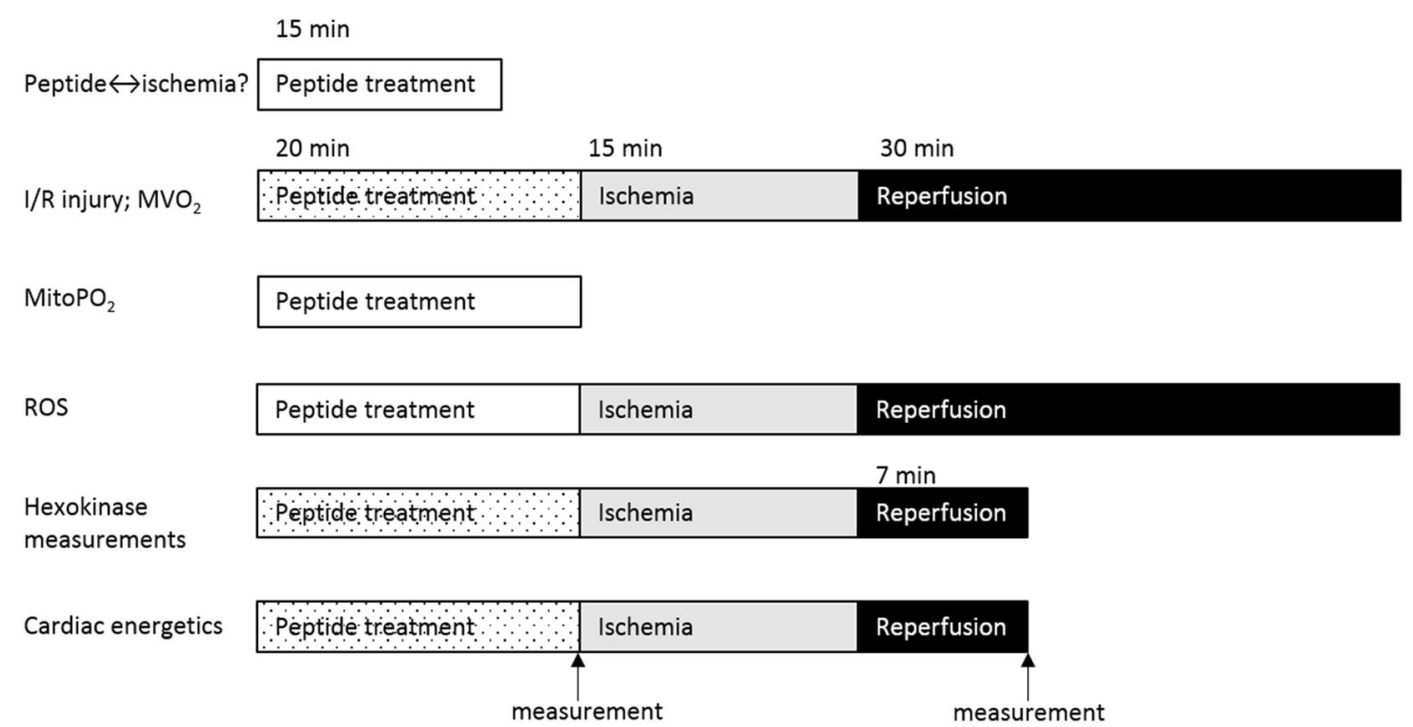

Fig. 1 Perfusion protocols used for peptide effects on ischemia development, I/R injury and cardiac oxygen consumption, mitochondrial $\mathrm{PO}_{2}$ within the intact heart, ROS production, mitochondria-hexokinase binding and cardiac energetic parameters (PCr, ATP). Hearts were perfused with saline, TAT-only or TAT-HK peptide for 15-20 min after which some hearts were exposed to 15-min

ischemia and 7- or 30-min reperfusion. I/R, cardiac energetics and hearts for hexokinase measurements were paced at $320 \mathrm{bpm}$ during peptide treatment (dotted). NADH experiments were performed during 15-min peptide treatment. Cardiac energetics was measured at the end of peptide treatment and at 7-min reperfusion

\section{Lactate production and NADH epifluorescence}

During the execution of the present study, new research suggested that high doses of TAT-HKII may induce ischemia in the intact heart [26]. Therefore, NADH fluorescence of the surface of the heart and effluent lactate were evaluated as direct indices of ischemia in the $1 \mu \mathrm{M}$ TAT-HKII group as described before [23] and compared to the control $(2.5 \mu \mathrm{M}$ TAT-HKII and $200 \mathrm{nM}$ TAT-only) values of that study.

\section{Ischemia/reperfusion experiments}

Rat hearts were perfused as described above. Hearts were paced at $320 \mathrm{bpm}$, and oxygen consumption $\left(\mathrm{MVO}_{2}\right)$ was measured using an oxygen sensor (World Precision Instruments) in the left pulmonary artery. After the 20-min stabilisation period, hearts were exposed to 20-min peptide treatment, followed by 15-min global no-flow ischemia and 30-min reperfusion. During ischemia, hearts were submerged in KHB gassed with $95 \% \mathrm{~N}_{2} / 5 \% \mathrm{CO}_{2}$, and pacing was stopped. During reperfusion, flow was slowly increased to initial flow within the first minute of reperfusion. Venous effluent was sampled at 5-, 10-, 20- and 30-min reperfusion for determination of lactate dehydrogenase (LDH) leakage as index of cell death.

\section{Mitochondrial oxygen tension}

Mitochondrial oxygen tension $\left(\right.$ mito $\left.\mathrm{PO}_{2}\right)$ was measured in a separate series of Langendorff perfused hearts using

protoporphyrin IX (PpIX) as described before [18, 19]. In short, rats were injected with ALA (5-aminolevulinic acid hydrochloride) $2 \mathrm{~h}$ before the heart was dissected to enhance PpIX. Langendorff perfusion was performed as described above, but without pacing of the heart. Hearts were exposed for $20 \mathrm{~min}$ to saline or $200 \mathrm{nM}$ TAT-HKII ( $n=5$ per group). PpIX was excited by a laser, and its delayed fluorescence was measured at 0-, 5-, 10-, 15- and 20-min peptide infusion.

\section{ROS measurements}

In a dedicated series of experiments for ROS measurements, Langendorff perfused rat hearts ( $n=6$ per group) were exposed to 20 -min treatment with $1 \mu \mathrm{M}$ TAT-only or $200 \mathrm{nM}$ TAT-HKII after which they were subjected to 15-min ischemia followed by $30-\mathrm{min}$ reperfusion. An additional small series $(n=3)$ examined saline effects on ROS. Relative changes in $\mathrm{O}_{2}$ levels were measured using DHE fluorescence as described before [2].

\section{Determination of mitochondrial hexokinase}

Rat hearts ( $n=6$ per group) were Langendorff perfused and exposed to saline, $1 \mu \mathrm{M}$ TAT-only or $200 \mathrm{nM}$ for $20 \mathrm{~min}$. Then, hearts were exposed to 15-min no-flow ischemia. After 7-min reperfusion, hearts were homogenised as described before [41]. The 7-min time-point was chosen, because at that time-point, the peak in ROS levels was observed. Hearts were minced and homogenized on ice in ice-cold homogenisation medium (0.25 M sucrose, 0.02 M HEPES and 
$1 \mathrm{mM}$ dithiothreitol, $\mathrm{pH}$ 7.4). Part of the homogenate was immediately centrifuged at $10,000 \mathrm{~g}$ for $10 \mathrm{~min}$ at $4{ }^{\circ} \mathrm{C}$. The pellet, representing the mitochondrial enriched fraction, was quickly frozen and stored at $-80{ }^{\circ} \mathrm{C}$ until western blotting and determination of $\mathrm{HK}$ activity. We have previously shown that just one $10,000 \mathrm{~g}$ centrifugation step results in a 2.5 times mitochondrial enrichment of the pellet as compared to whole homogenate [12]. Then, the mitochondrial enriched fraction was dissolved in 1-mL homogenisation medium with $50-\mu \mathrm{L}$ $10 \%$ Triton X-100 and 10- $\mu \mathrm{L} 100 \mathrm{mM}$ glucose-6-phosphate (to promote release of $\mathrm{HK}$ ), stirred and incubated $15 \mathrm{~min}$ at room temperature and sonificated two times for $5 \mathrm{~s}$. After centrifugation for $1 \mathrm{~min}$ at $10,000 \mathrm{~g}$, the amount of protein was determined in the supernatant using the Bradford protein assay.

\section{Western blot}

The amount of mtHKII was determined using western blot. For western blot, equal amounts of protein were loaded on a 4-12\% gradient gel (Biorad), electrophoresed and transferred to a polyvinylidene membrane [24]. Membranes were incubated overnight with the primary antibody against HKII (1:1000; Cell Signalling C64G5) and the mitochondrial marker COX IV (1:9000; Cell Signalling 4844). Immunoreactive bands were visualized by the Odyssey system and quantified using the Odyssey IR Manager. All samples were analysed on the same blot.

\section{Lactate dehydrogenase and hexokinase activity}

LDH activity in the effluent was determined as a measure of cell death, since other studies have shown a good correlation between LDH activity and TTC staining [20, 29]. It was determined spectrophotometrically by measuring NADH oxidation at $340 \mathrm{~nm}$ after addition of pyruvate in samples collected 5-, 10-, 20- and 30-min reperfusion at $25^{\circ} \mathrm{C}$.

$\mathrm{HK}$ activity was measured in mitochondrial enriched samples at $25{ }^{\circ} \mathrm{C}$ with glucose-6-phosphate dehydrogenase, glucose, ATP and NAD ${ }^{+}$, in the presence of rotenone $(1 \mu \mathrm{M})$ to inhibit NADH breakdown by oxidoreductase enzyme activity. Mitochondrial enriched samples were corrected for citrate synthase (CS), a mitochondrial marker. CS was determined at $25{ }^{\circ} \mathrm{C}$ using acetyl-CoA, oxaloacetate and dithionitrobenzoic acid, measuring the formation of thionitrobenzoic acid at $412 \mathrm{~nm}$.

\section{Cardiac energetics}

Cardiac energetics was measured in Langendorff perfused rat hearts ( $n=4-6$ per group). Hearts were exposed for $20 \mathrm{~min}$ to $1 \mu \mathrm{M}$ TAT-only or $200 \mathrm{nM}$ TAT-HKII. Thereafter, for one group, hearts were snap frozen in liquid nitrogen, weighed and stored at $-80{ }^{\circ} \mathrm{C}$ until analysis. Another group was exposed to 15 -min ischemia followed by 7 -min reperfusion, after which the hearts were frozen.

Hearts were freeze-dried overnight and dry weight was determined. ATP, PCr, creatinine $(\mathrm{Cr})$ and inorganic phosphate $(\mathrm{Pi})$ were determined as described by Fiolet et al. [9]. The phosphorylation potential $\left(\Delta \mathrm{G}_{\mathrm{ATP}}\right)$ was calculated from these values.

\section{Statistical analysis}

Data are presented as mean + SEM. Data were analysed by an independent $t$ test when there were two groups, or by ANOVA followed by a Dunnet's post hoc test with saline as control group when there were more than two groups. $\mathrm{MVO}_{2}, \mathrm{RPP}$ and $\mathrm{MVO}_{2} / \mathrm{RPP}$ at baseline were analysed by a two-way ANOVA for time and group, and Dunnet's post hoc test when applicable. $\mathrm{MitoPO}_{2}$ was tested using a paired $t$ test.

\section{Results}

\section{$1 \mu \mathrm{M}$ TAT-HKII causes ischemia}

In TAT-only and $200 \mathrm{nM}$ TAT-HKII-treated hearts, 15-min peptide treatment did not lead to an increase in NADH fluorescence (97 and 95\% from baseline, respectively) (Fig. 2a; published previously [23]). In addition, both treatments did not lead to lactate production (Fig. 2b). However, $1 \mu \mathrm{M}$ TAT-HKII treatment caused an increase in NADH fluorescence to $147 \%$ of baseline (Fig. 2a). This increase was significant when compared to TAT-only $(p<0.001)$. In addition, it non-significantly increased lactate production $(p=0.051)$ (Fig. 2b), and perfusion pressure was significantly increased to $112.6 \mathrm{mmHg}$ after 20 -min perfusion ( $p=0.006$ ) (Fig. 2c). These results indicate that $1 \mu \mathrm{M}$ TAT-HKII treatment causes ischemia. Because this makes it difficult to distinguish the effects of HKII translocation and ischemia, we decided to only report the treatment groups (saline, TAT-only, $200 \mathrm{nM}$ TATHKII) for which no signs of ischemia were detected.

\section{TAT-HKII decreases oxygen consumption but is without effect on cardiac economy before ischemia}

In both control groups, $\mathrm{MVO}_{2}$ was decreased to $96 \%$ of baseline at the end of peptide treatment (Fig. 3a). In the $200 \mathrm{nM}$ TAT-HKII group, this decrease was significantly larger $(88 \%$ of baseline; $p=0.004$ ). Cardiac function, as reflected by the rate-pressure-product, only showed a non-significant trend to decrease with TAT-HKII treatment (Fig. 3b). However, when corrected for RPP, $\mathrm{MVO}_{2}$ was not different in the $200 \mathrm{nM}$ TAT-HKII group when compared to control groups (Fig. 3c), indicating that cardiac economy was unaltered. 

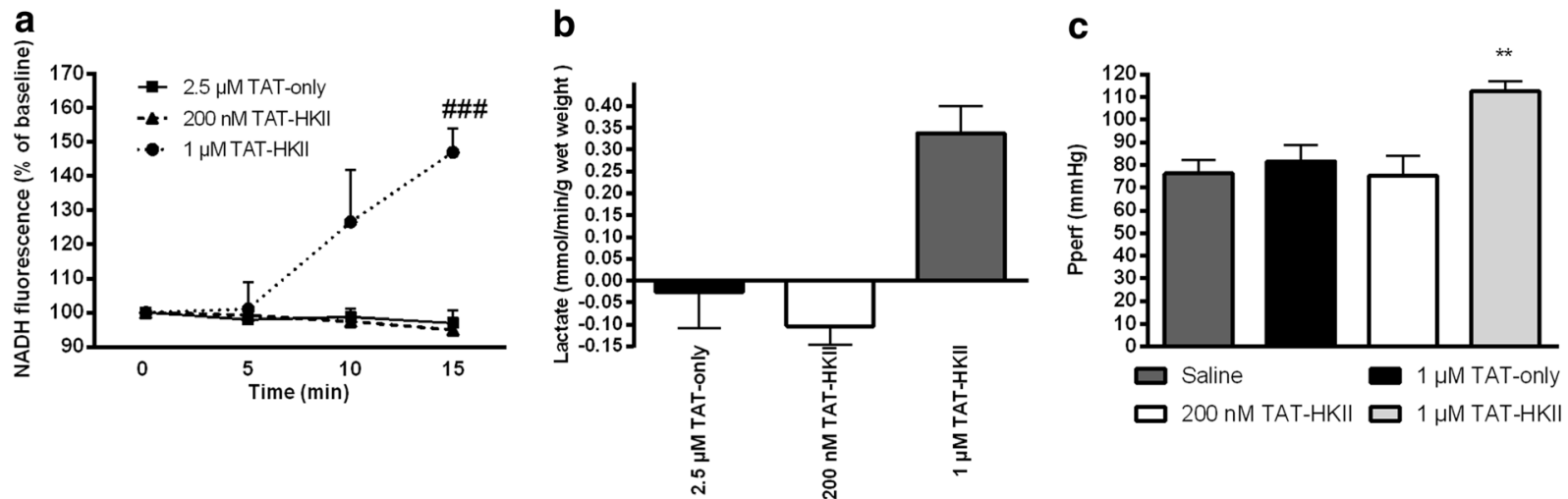

Fig. 2 Low-dose TAT-HKII caused no ischemia, whereas higher-dose TAT-HKII caused modest ischemia. Hearts were treated for $15 \mathrm{~min}$ with saline, $2.5 \mu \mathrm{M}$ TAT-only or $200 \mathrm{nM}$ or $1 \mu \mathrm{M}$ TAT-HKII. NADH fluorescence in the heart during the 15-min peptide treatment (a), lactate in effluent after 15-min peptide treatment (b) and perfusion

Next to $\mathrm{MVO}_{2}$, mitoPO $\mathrm{P}_{2}$ during peptide treatment was measured in a different set of experiments. Workload in these hearts was comparable to paced hearts (mean heart rate $277 \mathrm{bpm}$ and RPP 35,384 bpm * mmHg). At baseline, there were no differences in mito $\mathrm{PO}_{2}$ between groups (27.3 and $25.1 \mathrm{mmHg}$ for saline and $200 \mathrm{nM}$ TAT-HKII, respectively). TAT-HKII peptide treatment was not associated with a change in mean mitoPO $\mathrm{O}_{2}$ (Fig. 3d), supporting our previous results

pressure Pperf at the end of peptide treatment (c). ${ }^{\# \#} p<0.001$ vs $2.5 \mu$ M TAT-only; $* * p<0.01$ vs saline. Mean + SEM, $n=3-4$ for a and $\mathbf{b}, n=5-7$ for $\mathbf{c}$. Data for $2.5 \mu \mathrm{M}$ TAT-only and $200 \mathrm{nM}$ TAT-HKII in $\mathbf{a}$ and $\mathbf{b}$ have been published before [23] and are provided to facilitate direct comparisons with the $1 \mu \mathrm{M}$ TAT-HKII peptide treatment

(Fig. 2) that $200 \mathrm{nM}$ TAT-HKII treatment was not associated with cardiac ischemia.

\section{A reduction in $\mathbf{m t H K I I}$ turns reversible $\mathrm{I} / \mathrm{R}$ injury in irreversible $\mathbf{I} / \mathbf{R}$ injury}

At the end of reperfusion, both control groups showed no signs of I/R injury. Cell death was not observed (Fig. 4a),
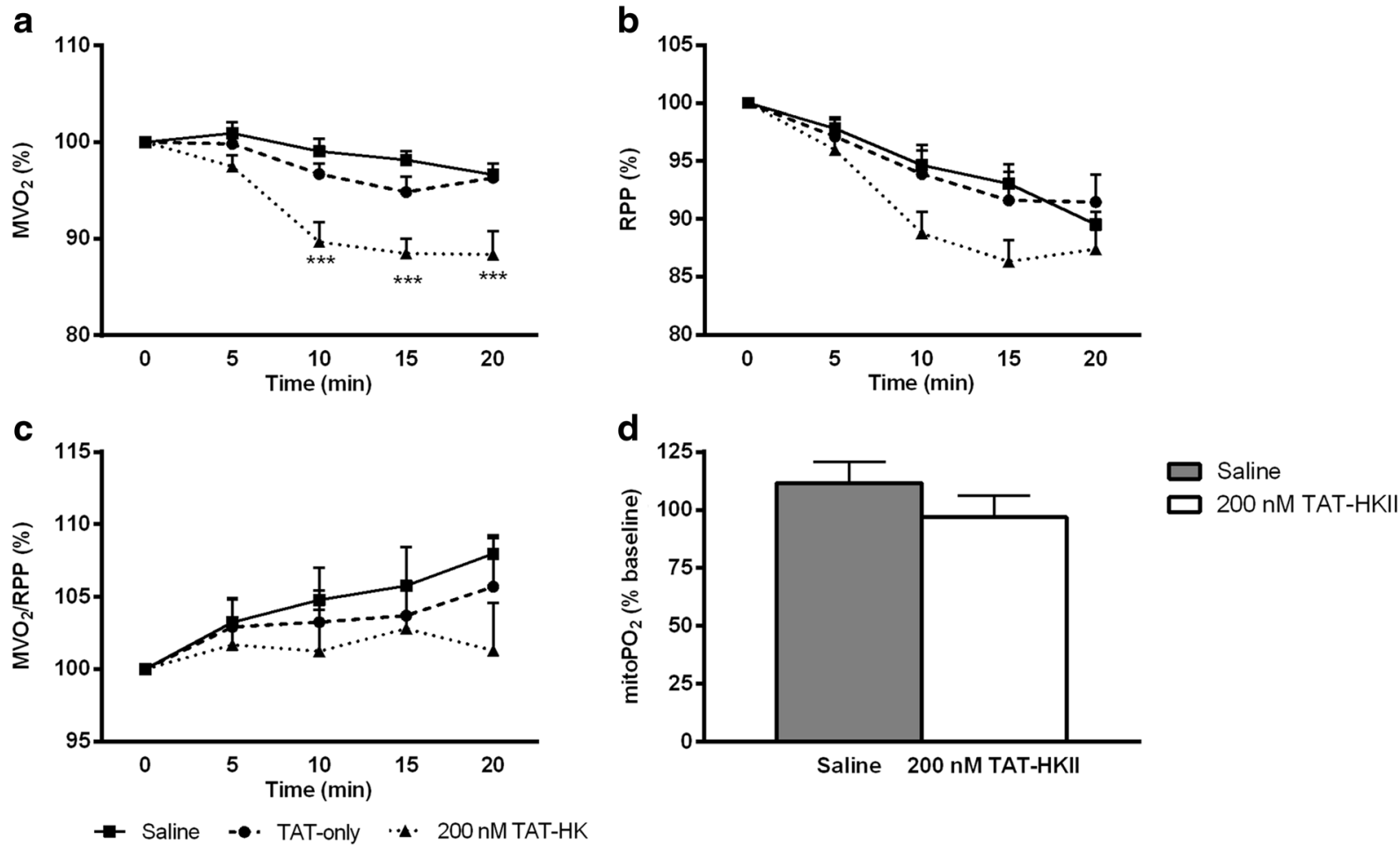

Fig. 3 TAT-HKII treatment decreased cardiac oxygen consumption but was without effect on myocardial economy before ischemia. Langendorff perfused rat hearts were exposed to $20 \mathrm{~min}$ saline, $1 \mu \mathrm{M}$ TAT-only or 200 nM TAT-HKII treatment. Cardiac oxygen consumption $\left(\mathrm{MVO}_{2}\right)(\mathbf{a})$, cardiac function (rate pressure product (RPP)) (b) and myocardial

economy $\left(\mathrm{MVO}_{2} / \mathrm{RPP}\right)$ (c) during Langendorff perfusion. Mitochondrial oxygen tension $\left(\mathrm{mitoPO}_{2}\right)$ after 20 -min peptide treatment as percentage of baseline (d). ${ }^{* *} p<0.01$ vs saline. Mean + SEM, $n=5-8$ per group 

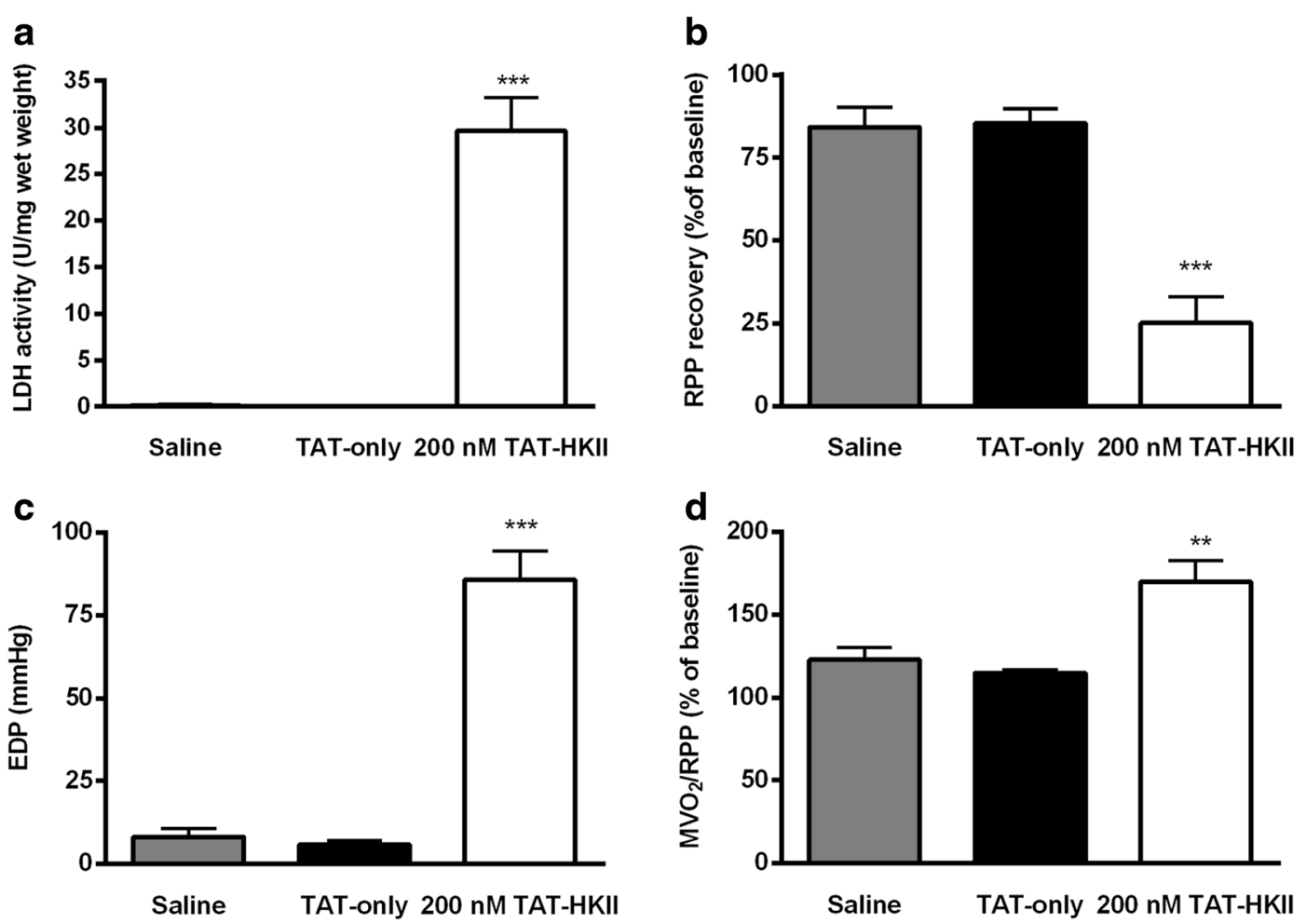

Fig. 4 A reduction in $\mathrm{mtHKII}$ turned reversible $\mathrm{I} / \mathrm{R}$ into irreversible $\mathrm{I} / \mathrm{R}$. Hearts were exposed to 20-min treatment with saline, $1 \mu \mathrm{M}$ TAT-only or $200 \mathrm{nM}$ TAT-HKII, followed by $15-\mathrm{min}$ ischemia and 30 -min reperfusion. Total lactate dehydrogenase (LDH) activity released during

RPP returned to 84 and $85 \%$ of baseline (Fig. 4b) and enddiastolic pressure (EDP) returned to 8 and $6 \mathrm{mmHg}$ (Fig. 4c) for the saline and $1 \mu \mathrm{M}$ TAT-only group, respectively. When HKII was translocated from the mitochondria, extensive I/R injury was observed: hearts had a pronounced significant rise in LDH release $(29.7 \mu \mathrm{mol} / \mathrm{g}$ heart weight/30-min reperfusion; $p<0.001$ ), cardiac recovery was reduced to $25 \%$ and EDP rose to $86 \mathrm{mmHg}$ (both $p<0.001$ ). In addition, TATHKII treatment decreased cardiac economy at end reperfusion, as $\mathrm{MVO}_{2} / \mathrm{RPP}$ was significantly higher in TAT-HKIIexposed group ( $p=0.001$ ) (Fig. 4d), indicating uncoupling between oxygen consumption and force development.

\section{TAT-HKII increases ROS}

Shown in Fig. 5a are the ROS levels indexed by normalized DHE fluorescence in TAT-control and TAT-HKII-treated hearts before and during the I/R intervention. Also, quantified are the ROS levels at the end of baseline, the end of ischemia and the peak levels during early reperfusion (Fig. 5b). Before ischemia, there were no differences in ROS levels between groups. In contrast, TAT-HKII-treated hearts exhibited significantly greater increase in ROS levels at $15 \mathrm{~min}$ of ischemia (by $63 \% ; p=0.039$ ) and at the peak during reperfusion (by $96 \% ; p=0.04)$ compared to control hearts. Of note, the difference in peak ROS levels between both groups was most

30-min reperfusion (a), rate pressure product (RPP) (b), end-diastolic pressure (EDP) (c) and cardiac oxygen consumption $\left(\mathrm{MVO}_{2}\right) / \mathrm{RPP}(\mathbf{d})$ determined at 30 -min reperfusion. $* * p<0.01$, $* * * p<0.001$ vs saline. Mean + SEM, $n=5-7$ per group

pronounced during the reperfusion phase. No differences between the TAT control group and a saline group $(n=3)$ were observed (data not shown).

\section{TAT-HKII translocates HKII from mitochondria}

At 7 min of reperfusion, we determined the amount of HKII in our mitochondria-enriched fraction (Fig. 6a, b). TAT-HKII treatment significantly reduced mtHKII binding with $26 \%$ compared to saline $(p=0.03)$. Additionally, HK activity confirmed the results observed by western blot (Fig. 6c). HK/CS activity, indicating both HKI and HKII activity, was reduced $13 \%$ in animals treated with $200 \mathrm{nM}$ TAT-HKII $(p=0.08)$. Knowing that HKI and HKII contribute approximately $50 \%$ to cardiac HK activity [34], the $13 \%$ of total HK activity then translates in a $26 \%$ reduction of HKII activity, commensurate with the $26 \%$ HKII protein reduction.

\section{TAT-HKII increased PCr before ischemia}

Twenty-minute treatment with $200 \mathrm{nM}$ TAT-HKII significantly increased $\mathrm{PCr}$ when compared to TAT-only treatment (26.7 vs $21.5 \mu \mathrm{mol} / \mathrm{g}$ dry weight, respectively; $p=0.02$ ) (Fig. 7a). In addition, $200 \mathrm{nM}$ TAT-HKII non-significantly increased $\mathrm{PCr} /$ ATP ratio, a clinical indicator for the energy state of the heart, from 0.85 to 1.05 ( $p=0.08)$ (Fig. 7e). No effects were 

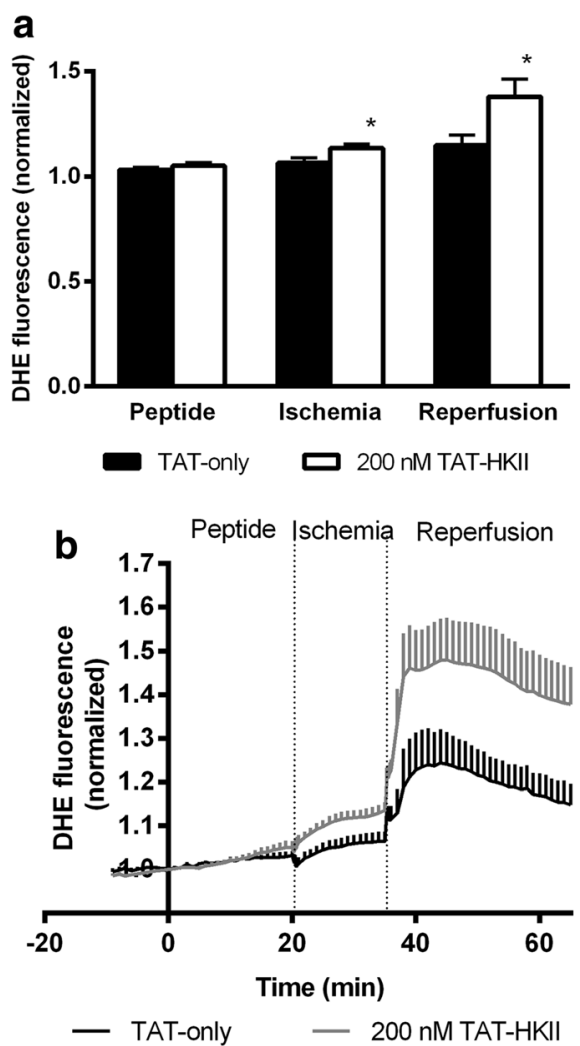

Fig. 5 TAT-HKII treatment increased ROS production during ischemia and reperfusion. ROS levels before ischemia (peptide treatment) and during the I/R intervention (a), and ROS levels at the end of peptide treatment, at the end of ischemia and at the peak during early reperfusion (b). $* p<0.05$ vs TAT-only. Mean + SEM, $n=6$ per group

observed on ATP (Fig. 7c), inorganic phosphate (Pi), creatine or free energy of ATP hydrolysis $\left(\Delta \mathrm{G}_{\mathrm{ATP}}\right)$ (data not shown).

In contrast to the effects of peptide treatment before ischemia, after ischemia $\mathrm{PCr}$ and $\mathrm{PCr} / \mathrm{ATP}$ were significantly lower with TAT-HKII treatment when compared to control $(p=0.02$ and $p=0.01$, respectively) (Fig. 7b, f). Also, after ischemia, no differences in ATP were observed (Fig. 7d).

\section{Discussion}

The results of our study show that a loss of mtHKII can mediate the transition from non-injurious reversible $\mathrm{I} / \mathrm{R}$ into one that is injurious, suggesting that the amount of HKII bound to mitochondria is a determinant of when $\mathrm{I} / \mathrm{R}$ becomes damaging. This loss of mtHKII improved cardiac energetics and reduced oxygen consumption before $\mathrm{I} / \mathrm{R}$, negating the possibility that reduced $\mathrm{mtHKII}$ caused increased cardiac I/R injury due to impaired energetics or increased mitochondrial respiration. In contrast, decreasing mtHKII causes increased ROS production during the ischemia and reperfusion period, suggesting the involvement of mtHKII in I/R-induced ROS production and that increased ROS may be the trigger for injury to start occurring.

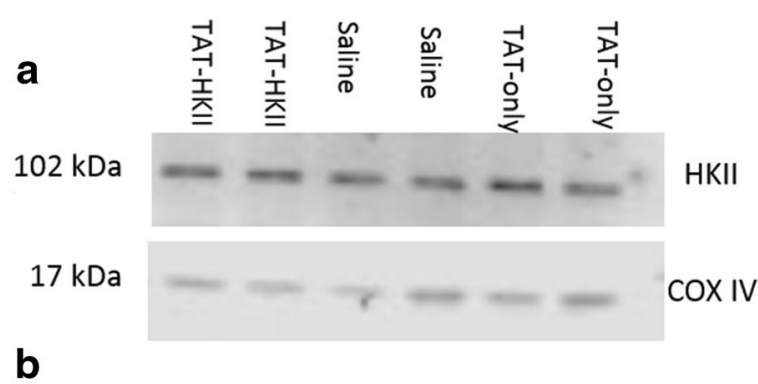

b

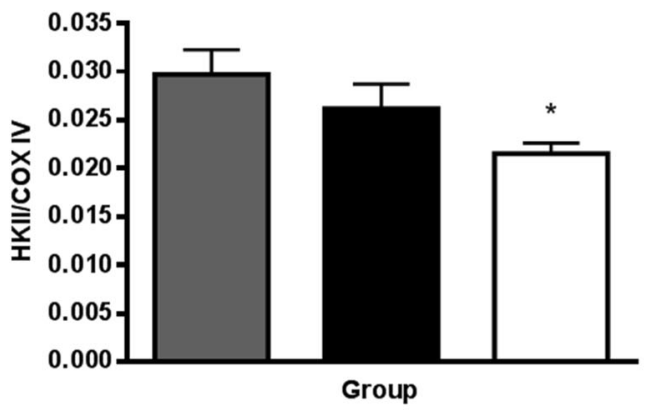

C

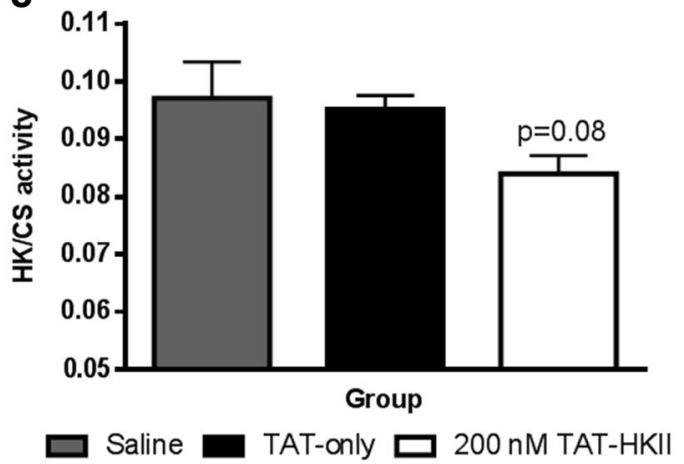

Fig. 6 TAT-HKII treatment reduced mitochondrial bound hexokinase. Hearts were treated for 20 min with saline, $1 \mu \mathrm{M}$ TAT-only or $200 \mathrm{nM}$ TAT-HKII, exposed to 15 -min ischemia and 7-min reperfusion. Representative images of the western blots (a), semi-quantitative amount of hexokinase II (HKII)/COX IV bound to the mitochondria as determined by western blot (b) and HK activity corrected for citrate synthase (CS) activity at 7 -min reperfusion (c). $* p<0.05$ vs saline. Mean + SEM, $n=6$ per group

Previous studies have shown that a reduction in mtHKII can exacerbate I/R injury $[28,33,39]$. The amount of $\mathrm{mtKII}$ has been shown to correlate with infarct size [28], and a reduction in mtHKII has been shown to block IPC [33], decrease cardiac function and alter cardiac remodelling [39]. In addition, cardioprotective interventions, like insulin, morphine and IPC, are associated with an increase in mtHKII [41]. In this study, we observed that a reduction in mtHKII causes cell death, increases EDP and reduces RPP after 15min ischemia. Factors are not affected in control hearts. The reduction observed in RPP in control hearts can be considered as normal deterioration over time in a Langendorff system [42]. This is the first study showing that a reduction in mtHKII can also turn a non-injurious episode of 15 -min ischemia into an injurious ischemia. 

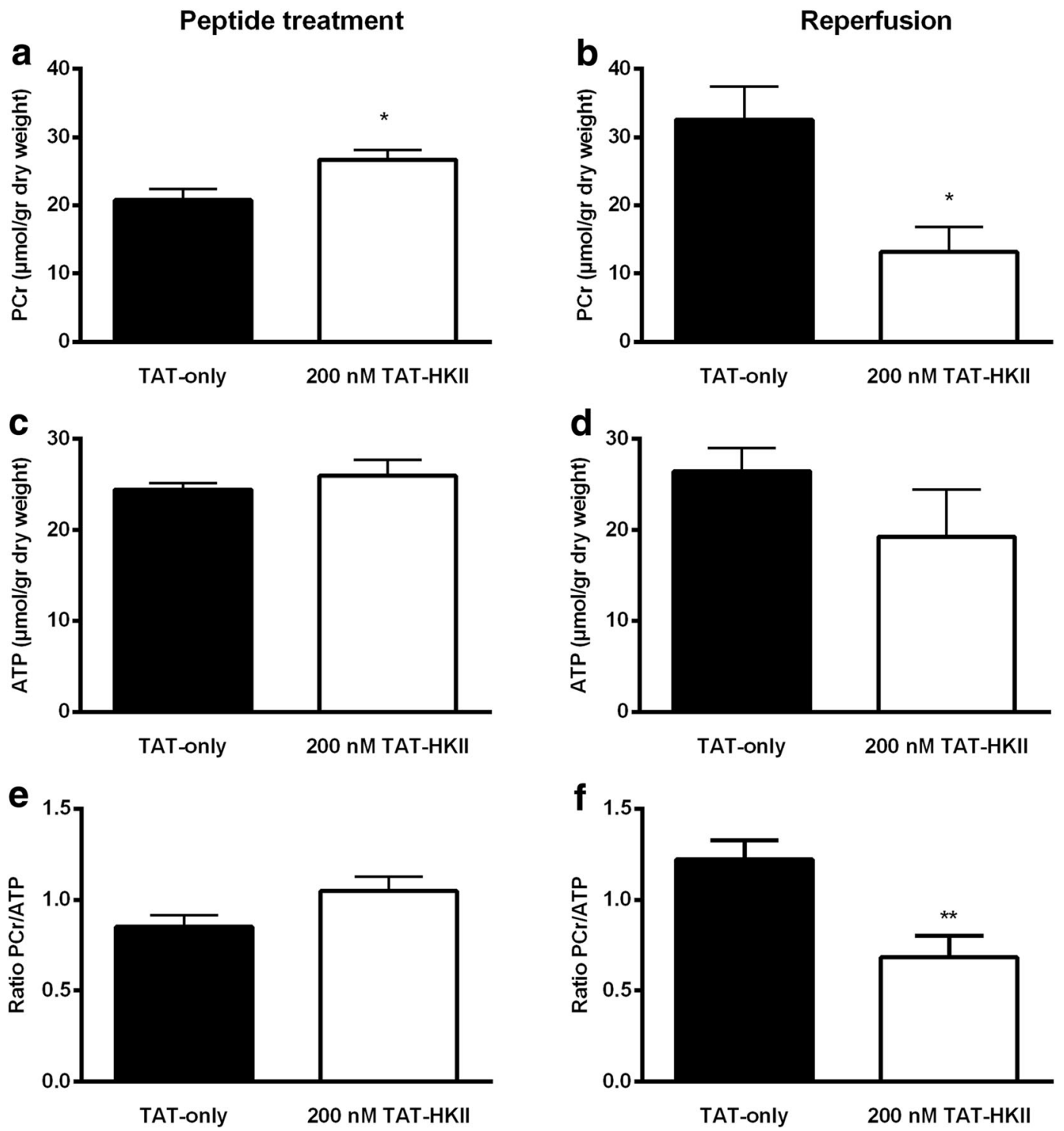

Fig. 7 TAT-HKII increases $\mathrm{PCr}$ before ischemia, but lowers $\mathrm{PCr}$ after ischemia. Hearts were treated for 20 min with $1 \mu$ M TAT-only or $200 \mathrm{nM}$ TAT-HKII. Energetics were measured at the end of peptide treatment (baseline) or after 15-min ischemia and 7-min reperfusion.

This switch from non-injurious to injurious ischemia might have been caused by an increase in ROS production during ischemia and reperfusion after HKII translocation. Multiple studies have shown that a reduction in mtHKII increases ROS production $[7,32,40]$, while an increase in mtHKII can reduce ROS $[25,37]$. ROS is an important factor in opening of the mitochondrial permeability transition pore (MPTP) [13]. Dimerization of $\mathrm{F}_{0} \mathrm{~F}_{1}$ ATPase in the mitochondrial membrane has recently been proposed as molecular identity of the MPTP [11]. The MPTP opens when matrix $\mathrm{Ca}^{2+}$ and ROS levels are high [13]. When the MPTP opens, molecules $<1.5 \mathrm{kDa}$ can passage the mitochondrial membrane, finally leading to cell death. The increase in ROS production after HKII translocation might have caused increased MPTP opening and thereby
Phosphocreatine $(\mathrm{PCr})(\mathbf{a}, \mathbf{b}), \operatorname{ATP}(\mathbf{c}, \mathbf{d})$ and ratio $\mathrm{PCr} / \mathrm{ATP}(\mathbf{e}, \mathbf{f})$ at the end of 20-min treatment $(\mathbf{a}, \mathbf{c}, \mathbf{e})$ and after ischemia $(\mathbf{b}, \mathbf{d}, \mathbf{f}) . * p<0.05$, $* * p<0.01$ vs TAT-only at the same time-point. Mean + SEM, $n=4-6$ per group

increased cell death. This study provides evidence that also after a short period of ischemia, a reduction in mtHKII causes an increase in ROS and might thereby increase cardiac cell death.

ROS may be increased during ischemia and reperfusion after mtHKII detachment because cytochrome $\mathrm{c}$ (cyt $\mathrm{c}$ ) is released from the intermembrane space. Previously, we showed that cytosolic cyt $\mathrm{c}$ is significantly higher in reperfused hearts after treatment with $200 \mathrm{nM}$ TAT-HKII [33]. Cyt $\mathrm{c}$ is a powerful superoxide scavenger present in the inner mitochondrial membrane. MtHKII forms mitochondrial contact sites with other proteins. Disruption of HKII from these contact sites might increase permeability of the OMM, as it does in reconstituted vesicles [1]. A translocation of HKII from the mitochondria, in combination with increased $\mathrm{Ca}^{2+}$ levels, disrupts contact sites 
and increases permeability of the OMM causing cyt $\mathrm{c}$ to be released $[27,33]$. This will result in increased ROS.

Recently, it has become clear that respiratory inhibition is a nodal point for several cardioprotective interventions (nitrosylation, IPC, hypothermia, respiratory inhibitors) through which protection against I/R injury is conferred. Previous research also indirectly indicated that increasing $\mathrm{mtHK}$ would slow down mitochondrial metabolism [12, 22, 42, 43]. We therefore anticipated that reduced mtHKII would result in increased oxygen consumption and thereby contribute to increased I/R injury. However, our results during baseline clearly show this not to be the case: TAT-HKII actually decreased $\mathrm{MVO}_{2}$ in the glucose-only perfused rat heart. However, since cardiac function also decreased, no changes in cardiac economy were observed. Whether similar effects can be observed in hearts perfused with different substrates will have to await further studies. In addition, our results negate the possibility that the transition to injurious I/R injury with TAT-HKII peptide treatment is mediated through increased mitochondrial respiration. The decreased $\mathrm{MVO}_{2}$ during baseline and the increased economy after I/R might indicate mitochondrial uncoupling.

The results also exclude a role for impaired energetics as mechanism provoking injurious $\mathrm{I} / \mathrm{R}$ injury upon loss of $\mathrm{mtHKII}$. The increased $\mathrm{PCr}$ at baseline is likely a consequence (secondary effect) of the reduction in cardiac oxygen consumption: the heart's energy turnover is decreased, resulting in a more energetic state of the heart $[8,38]$. In addition, the lower PCr levels after ischemia are probably not caused by a direct effect of the reduction in $\mathrm{mtHKII}$, but indirectly by cell death in these hearts.

During reperfusion, $\mathrm{PCr}$ in control hearts is higher than at baseline. It is known that during reversible ischemia, $\mathrm{PCr}$ levels decrease and quickly recover during reperfusion to levels higher than before ischemia. This phenomenon is known as 'PCr overshoot' [14]. The presence of PCr overshoot is another sign that the 15-min ischemia did not lead to irreversible damage in control hearts [36].

\section{Methodological considerations}

In this study, we planned to use two different concentrations of the TAT-HKII peptide. A low dose of $200 \mathrm{nM}$ that was previously shown by us to decrease mtHKII without causing ischemia $[23,33]$. The higher dose of $1 \mu \mathrm{M}$ was chosen to study larger effects of a loss of mtHKII. However, after start of the experiments, Pasdois et al. suggested that the TAT-HKII peptide impairs vascular function and that some of its effects may be mediated by vasoconstriction of the coronary vasculature and developing ischemia rather than dissociation of HKII from the mitochondria [26]. We showed that the low dose of $200 \mathrm{nM}$ TAT-HKII did not cause any ischemia and that effects observed by higher doses of the TAT-HKII peptide cannot be explained by ischemia alone [23]. We did, however, observe that $2.5 \mu \mathrm{M}$ TAT-HKII increased NADH epifluorescence and increased lactate production, indicating that it causes minor ischemic damage. To exclude a role of ischemia in this study, we also measured lactate and $\mathrm{NADH}$ epifluorescence after administering $1 \mu \mathrm{M}$ TAT-HKII, and we observed an increase in both parameters. Because it would be difficult to separate the effects caused by the HKII translocation and the ischemia caused by the peptide, we decided to continue with the 200 nM TAT-HKII dose only.

The protocol used to isolate mitochondria does not lead to isolation of a pure mitochondrial fraction, but a mitochondrial enriched fraction. A more elaborate isolation protocol would have led to $\mathrm{HK}$ dissociation from the mitochondria [16, 31]. $\mathrm{HK}$ in the heart is either located at the mitochondria or in the cytosol [4, 35], and therefore, the use of a more elaborate protocol was not necessary.

Finally, this study only looked at necrotic cell death and not apoptosis, since its role is only significant after a longer period of ischemia and/or reperfusion [10, 17]. In acute $\mathrm{I} / \mathrm{R}$ injury, necrosis has been observed as the main contributor to myocardial cell death $[15,17]$.

\section{Conclusions}

The present study shows that the loss of mtHKII can be a master switch turning reversible ischemia into irreversible ischemia. This loss of mtHKII is associated with increased ROS production after ischemia, but not with increased respiratory activity or decreased cardiac energetics at baseline.

Acknowledgements This study was supported by the Dutch Heart Foundation to CJZ (NHS2010B011).

Compliance with ethical standards

Data availability All data is available from the authors upon request.

Open Access This article is distributed under the terms of the Creative Commons Attribution 4.0 International License (http:// creativecommons.org/licenses/by/4.0/), which permits unrestricted use, distribution, and reproduction in any medium, provided you give appropriate credit to the original author(s) and the source, provide a link to the Creative Commons license, and indicate if changes were made.

\section{References}

1. Beutner G, Ruck A, Riede B, Brdiczka D (1998) Complexes between porin, hexokinase, mitochondrial creatine kinase and adenylate translocator display properties of the permeability transition pore. Implication for regulation of permeability transition by the kinases. Biochim Biophys Acta. doi:10.1016/s0005-2736(97)00175-2 
2. Biary N, Xie C, Kauffman J, Akar FG (2011) Biophysical properties and functional consequences of reactive oxygen species (ROS)induced ROS release in intact myocardium. J Physiol. doi:10.1113 /jphysiol.2011.214239

3. Burwell LS, Nadtochiy SM, Brookes PS (2009) Cardioprotection by metabolic shut-down and gradual wake-up. J Mol Cell Cardiol. doi:10.1016/j.yjmcc.2009.02.026

4. Calmettes G, John SA, Weiss JN, Ribalet B (2013) Hexokinasemitochondrial interactions regulate glucose metabolism differentially in adult and neonatal cardiac myocytes. J Gen Physiol. doi:10.1085/jgp.201310968

5. Chiara F, Castellaro D, Marin O, Petronilli V, Brusilow WS, Juhaszova M, Sollott SJ, Forte M, Bernardi P, Rasola A (2008) Hexokinase II detachment from mitochondria triggers apoptosis through the permeability transition pore independent of voltagedependent anion channels. PLoS One. doi:10.1371/journal. pone. 0001852

6. Colombini M (2004) VDAC: the channel at the interface between mitochondria and the cytosol. Mol Cell Biochem 256-257:107-115

7. da-Silva WS, Gomez-Puyou A, de Gomez-Puyou MT, MorenoSanchez R, De Felice FG, de Meis L, Oliveira MF, Galina A (2004) Mitochondrial bound hexokinase activity as a preventive antioxidant defense: steady-state ADP formation as a regulatory mechanism of membrane potential and reactive oxygen species generation in mitochondria. J Biol Chem. doi:10.1074/jbc. M403835200

8. Eijgelshoven $\mathrm{MH}$, van Beek JH, Mottet I, Nederhoff MG, van Echteld CJ, Westerhof N (1994) Cardiac high-energy phosphates adapt faster than oxygen consumption to changes in heart rate. Circ Res 75:751-759

9. Fiolet JW, Baartscheer A, Schumacher CA, Coronel R, ter Welle HF (1984) The change of the free energy of ATP hydrolysis during global ischemia and anoxia in the rat heart. Its possible role in the regulation of transsarcolemmal sodium and potassium gradients. $\mathrm{J}$ Mol Cell Cardiol 16:1023-1036

10. Freude B, Masters TN, Robicsek F, Fokin A, Kostin S, Zimmermann R, Ullmann C, Lorenz-Meyer S, Schaper J (2000) Apoptosis is initiated by myocardial ischemia and executed during reperfusion. J Mol Cell Cardiol. doi:10.1006/jmcc.1999.1066

11. Giorgio V, von Stockum S, Antoniel M, Fabbro A, Fogolari F, Forte M, Glick GD, Petronilli V, Zoratti M, Szabo I, Lippe G, Bernardi P (2013) Dimers of mitochondrial ATP synthase form the permeability transition pore. Proc Natl Acad Sci U S A. doi:10.1073 /pnas. 1217823110

12. Gurel E, Smeele KM, Eerbeek O, Koeman A, Demirci C, Hollmann MW, Zuurbier CJ (2009) Ischemic preconditioning affects hexokinase activity and HKII in different subcellular compartments throughout cardiac ischemia-reperfusion. J Appl Physiol (1985). doi:10.1152/japplphysiol.90537.2008

13. Halestrap AP (2010) A pore way to die: the role of mitochondria in reperfusion injury and cardioprotection. Biochem Soc Trans. doi:10.1042/bst0380841

14. Kaplan LJ, Bellows CF, Carter S, Blum H, Whitman GJ (1995) The phosphocreatine overshoot occurs independent of myocardial work. Biochimie 77:245-248

15. Kung G, Konstantinidis K, Kitsis RN (2011) Programmed necrosis, not apoptosis, in the heart. Circ Res. doi:10.1161 /circresaha.110.225730

16. Lynch RM, Fogarty KE, Fay FS (1991) Modulation of hexokinase association with mitochondria analyzed with quantitative threedimensional confocal microscopy. J Cell Biol 112:385-395

17. McCully JD, Wakiyama H, Hsieh YJ, Jones M, Levitsky S (2004) Differential contribution of necrosis and apoptosis in myocardial ischemia-reperfusion injury. Am J Physiol Heart Circ Physiol. doi:10.1152/ajpheart.00935.2003
18. Mik EG, Ince C, Eerbeek O, Heinen A, Stap J, Hooibrink B, Schumacher CA, Balestra GM, Johannes T, Beek JF, Nieuwenhuis AF, van Horssen P, Spaan JA, Zuurbier CJ (2009) Mitochondrial oxygen tension within the heart. J Mol Cell Cardiol. doi:10.1016/j.yjmcc.2009.02.002

19. Mik EG, Stap J, Sinaasappel M, Beek JF, Aten JA, van Leeuwen TG, Ince C (2006) Mitochondrial PO2 measured by delayed fluorescence of endogenous protoporphyrin IX. Nat Methods. doi:10.1038/nmeth940

20. Morrison RR, Jones R, Byford AM, Stell AR, Peart J, Headrick JP, Matherne GP (2000) Transgenic overexpression of cardiac A(1) adenosine receptors mimics ischemic preconditioning. Am J Physiol Heart Circ Physiol 279:H1071-H1078

21. Nadtochiy SM, Urciuoli W, Zhang J, Schafer X, Munger J, Brookes PS (2015) Metabolomic profiling of the heart during acute ischemic preconditioning reveals a role for SIRT1 in rapid cardioprotective metabolic adaptation. J Mol Cell Cardiol. doi:10.1016/j. yjmcc.2015.09.008

22. Nederlof R, Eerbeek O, Hollmann MW, Southworth R, Zuurbier CJ (2014) Targeting hexokinase II to mitochondria to modulate energy metabolism and reduce ischaemia-reperfusion injury in heart. Br J Pharmacol. doi:10.1111/bph.12363

23. Nederlof R, Xie C, Eerbeek O, Koeman A, Milstein DM, Hollmann MW, Mik EG, Warley A, Southworth R, Akar FG, Zuurbier CJ (2013) Pathophysiological consequences of TAT-HKII peptide administration are independent of impaired vascular function and ensuing ischemia. Circ Res. doi:10.1161/CIRCRESAHA.112.274308

24. Oei GT, Huhn R, Heinen A, Hollmann MW, Schlack WS, Preckel B, Weber NC (2012) Helium-induced cardioprotection of healthy and hypertensive rat myocardium in vivo. Eur J Pharmacol. doi:10.1016/j.ejphar.2012.03.045

25. Ong SG, Lee WH, Theodorou L, Kodo K, Lim SY, Shukla DH, Briston T, Kiriakidis S, Ashcroft M, Davidson SM, Maxwell PH, Yellon DM, Hausenloy DJ (2014) HIF-1 reduces ischaemiareperfusion injury in the heart by targeting the mitochondrial permeability transition pore. Cardiovasc Res. doi:10.1093/cvr/cvu172

26. Pasdois P, Parker JE, Griffiths EJ, Halestrap AP (2013) Hexokinase II and reperfusion injury: TAT-HK2 peptide impairs vascular function in Langendorff-perfused rat hearts. Circ Res. doi:10.1161 /CIRCRESAHA.112.274233

27. Pasdois P, Parker JE, Griffiths EJ, Halestrap AP (2011) The role of oxidized cytochrome $\mathrm{c}$ in regulating mitochondrial reactive oxygen species production and its perturbation in ischaemia. Biochem $\mathrm{J}$. doi:10.1042/bj20101957

28. Pasdois P, Parker JE, Halestrap AP (2013) Extent of mitochondrial hexokinase II dissociation during ischemia correlates with mitochondrial cytochrome $\mathrm{c}$ release, reactive oxygen species production, and infarct size on reperfusion. J Am Heart Assoc. doi:10.1161/jaha.112.005645

29. Peart J, Headrick JP (2003) Adenosine-mediated early preconditioning in mouse: protective signaling and concentration dependent effects. Cardiovasc Res 58:589-601

30. Pell VR, Chouchani ET, Frezza C, Murphy MP, Krieg T (2016) Succinate metabolism: a new therapeutic target for myocardial reperfusion injury. Cardiovasc Res. doi:10.1093/cvr/cvw100

31. Picard M, Taivassalo T, Gouspillou G, Hepple RT (2011) Mitochondria: isolation, structure and function. J Physiol. doi:10.1113/jphysiol.2011.212712

32. Santiago AP, Chaves EA, Oliveira MF, Galina A (2008) Reactive oxygen species generation is modulated by mitochondrial kinases: correlation with mitochondrial antioxidant peroxidases in rat tissues. Biochimie. doi:10.1016/j.biochi.2008.06.013

33. Smeele KM, Southworth R, Wu R, Xie C, Nederlof R, Warley A, Nelson JK, van Horssen P, van den Wijngaard JP, Heikkinen S, Laakso M, Koeman A, Siebes M, Eerbeek O, Akar FG, Ardehali H, Hollmann MW, Zuurbier CJ (2011) Disruption of hexokinase II- 
mitochondrial binding blocks ischemic preconditioning and causes rapid cardiac necrosis. Circ Res. doi:10.1161/circresaha.111.244962

34. Smeele KM, ter Horst LH, Koeman A, Heikkinen S, Laakso M, Weber NC, Hollmann MW, Zuurbier CJ (2011) The effect of standard chow and reduced hexokinase II on growth, cardiac and skeletal muscle hexokinase and low-flow cardiac ischaemia-reperfusion injury. Lab Anim. doi:10.1258/la.2011.010096

35. Southworth R, Davey KAB, Warley A, Garlick PB (2007) A reevaluation of the roles of hexokinase I and II in the heart. Am J Physiol Heart Circ Physiol. doi:10.1152 /ajpheart.00664.2006

36. Straeter-Knowlen IM, Butterworth EJ, Buchthal SD, Hollander JA, Caulfield JB, Jennings RB, Evanochko WT (2002) PCr overshoot': a study of the duration in canine myocardium. NMR Biomed 15: 52-59

37. Sun L, Shukair S, Naik TJ, Moazed F, Ardehali H (2008) Glucose phosphorylation and mitochondrial binding are required for the protective effects of hexokinases I and II. Mol Cell Biol. doi:10.1128/mcb.00224-07

38. Wilson DF (2013) Regulation of cellular metabolism: programming and maintaining metabolic homeostasis. J Appl Physiol 1985. doi:10.1152/japplphysiol.00894.2013
39. Wu R, Smeele KM, Wyatt E, Ichikawa Y, Eerbeek O, Sun L, Chawla K, Hollmann MW, Nagpal V, Heikkinen S, Laakso M, Jujo K, Wasserstrom JA, Zuurbier CJ, Ardehali H (2011) Reduction in hexokinase II levels results in decreased cardiac function and altered remodeling after ischemia/reperfusion injury. Circ Res. doi:10.1161/circresaha.110.223115

40. Wu R, Wyatt E, Chawla K, Minh T, Ghanefar M, Laakso M, Epting CL, Ardehali H (2012) Hexokinase II knockdown results in exaggerated cardiac hypertrophy via increased ROS production. EMBO Mol Med. doi:10.1002/emmm.201200240

41. Zuurbier CJ, Eerbeek O, Meijer AJ (2005) Ischemic preconditioning, insulin, and morphine all cause hexokinase redistribution. Am J Physiol Heart Circ Physiol. doi:10.1152/ajpheart.01182.2004

42. Zuurbier CJ, Ince C (2002) Post-ischaemic changes in the response time of oxygen consumption to demand in the isolated rat heart are mediated partly by calcium and glycolysis. Pflugers Arch. doi:10.1007/s00424-001-0744-2

43. Zuurbier CJ, van Beek JH (1997) Mitochondrial response to heart rate steps in isolated rabbit heart is slowed after myocardial stunning. Circ Res 81:69-75 